\title{
Experimental analysis of pilot-based equalization for probabilistically shaped WDM systems with 256QAM/1024QAM
}

Yankov, Metodi Plamenov; Porto da Silva, Edson; Da Ros, Francesco; Zibar, Darko

Published in:

Optical Fiber Communication Conference 2017

Link to article, DOI:

10.1364/OFC.2017.W2A.48

Publication date:

2017

Document Version

Peer reviewed version

Link back to DTU Orbit

Citation (APA):

Yankov, M. P., Porto da Silva, E., Da Ros, F., \& Zibar, D. (2017). Experimental analysis of pilot-based equalization for probabilistically shaped WDM systems with 256QAM/1024QAM. In Optical Fiber Communication Conference 2017 [W2A.48] Optical Society of America (OSA). https://doi.org/10.1364/OFC.2017.W2A.48

\section{General rights}

Copyright and moral rights for the publications made accessible in the public portal are retained by the authors and/or other copyright owners and it is a condition of accessing publications that users recognise and abide by the legal requirements associated with these rights.

- Users may download and print one copy of any publication from the public portal for the purpose of private study or research.

- You may not further distribute the material or use it for any profit-making activity or commercial gain

- You may freely distribute the URL identifying the publication in the public portal 


\title{
Experimental analysis of pilot-based equalization for probabilistically shaped WDM systems with 256QAM/1024QAM
}

\author{
Metodi P. Yankov, Edson P. da Silva, Francesco Da Ros, and Darko Zibar \\ Department of Photonics Engineering, Technical University of Denmark, 2800 Kgs. Lyngby, Denmark \\ meya@fotonik.dtu.dk
}

\begin{abstract}
Pilot based equalization is studied in a 5x10 GBaud WDM transmission experiment. The equalization is independent of the modulation format and is demonstrated for 256/1024QAM with uniform and probabilistically optimized distribution using an optimized pilot insertion rate of $2-5 \%$.
\end{abstract}

OCIS codes: $060.1660,060.2330$.

\section{Introduction}

The advancement of coherent communications in recent years has enabled modulation formats with high spectral efficiency, such as high-order quadrature amplitude modulation (QAM), to become a major focus of the optical communications community. Such modulation formats, while theoretically sound, pose significant challenge for practical implementation due to the requirement for high optical signal-to-noise ratio (OSNR). Furthermore, optical communications are pushed to operate at lower OSNR and with higher forward error-correction (FEC) overhead in order to operate closer to the optimal OSNR/data rate trade-off (also known as the channel capacity in this context). Timedomain equalization in current receivers relies on the simple constant modulus algorithm (CMA) for QPSK constellations and its modification for QAM - the multi-modulus algorithm (MMA) [1]. The most popular MMA must first decide what amplitude each symbol should have, and then optimizes the equalizer w.r.t. the error between the actual received symbols and the so-defined radius [2]. This is also known as radius directed equalization (RDE). In order to operate near-optimally, the MMA requires that most of the decisions are correct, which is a strong requirement, especially in the lower OSNR/high FEC overhead operating points. Additionally, MMA also poses challenges for advanced modulation formats, e.g. probabilistic constellation shaping formats [3,5], in which case some of the radii are sparsely populated by the input sequence, thus deteriorating the MMA performance. In order to study the performance of such formats, modulation format- and SNR-independent equalization methods are required.

A pilot-based equalization method which meets the above requirements was used in a simulation [4] and experiment [5] of a probabilistically shaped QAM signal. Recently, similar method was proposed for multi-carrier 1 Tbps channels [6] and evaluated in optical back-to-back with up to 64QAM. In this paper, the method from $[4,5]$ is demonstrated and optimized experimentally w.r.t. the achievable information rate (AIR) in a 5x10 Gbaud wavelength division multiplexed (WDM) transmission system operating at 256/1024QAM with probabilistic shaping for up to $1600 \mathrm{~km}$.

\section{Pilot based CMA equalization and experimental setup}

In order to take advantage of the low-OSNR requirement of CMA equalization, the QAM sequence is interleaved with QPSK pilots, which are known at the receiver. A sketch of the system is given on the bottom of Fig. 1. The QPSK symbols appear one at a time with a certain period, $P-1$ (every $P$-th symbol is pilot), resulting in a pilot overhead (PO) of $1 /(P-1) \cdot 100 \%$. Furthermore, a short Zaddof-chu sequence with frequency- and phase-offset independent high correlation properties is used as a frame alignment sequence (FAS). At the receiver side, after alignment the pilot positions are identified. The equalization filter taps $\mathbf{H}_{\text {init }}$ are first estimated from a training sequence of QAM symbols, which is available at the receiver. At the first pilot position, $P$, the taps $\mathbf{H}_{\text {init }}$ are updated according to the CMA rule by estimating the error at that time and updating with a proper step $\mu$, which takes into account the distance $P$. The rest of the pilots are equalized in a similar manner. The process is repeated (in our case 20 times) until convergence. Following is linear interpolation between the taps at the pilot positions to estimate the taps $\mathbf{H}_{1}^{P-1}, \mathbf{H}_{P+1}^{2 P-1}, \ldots \mathbf{H}_{k P+1}^{(k+1) P}$, 


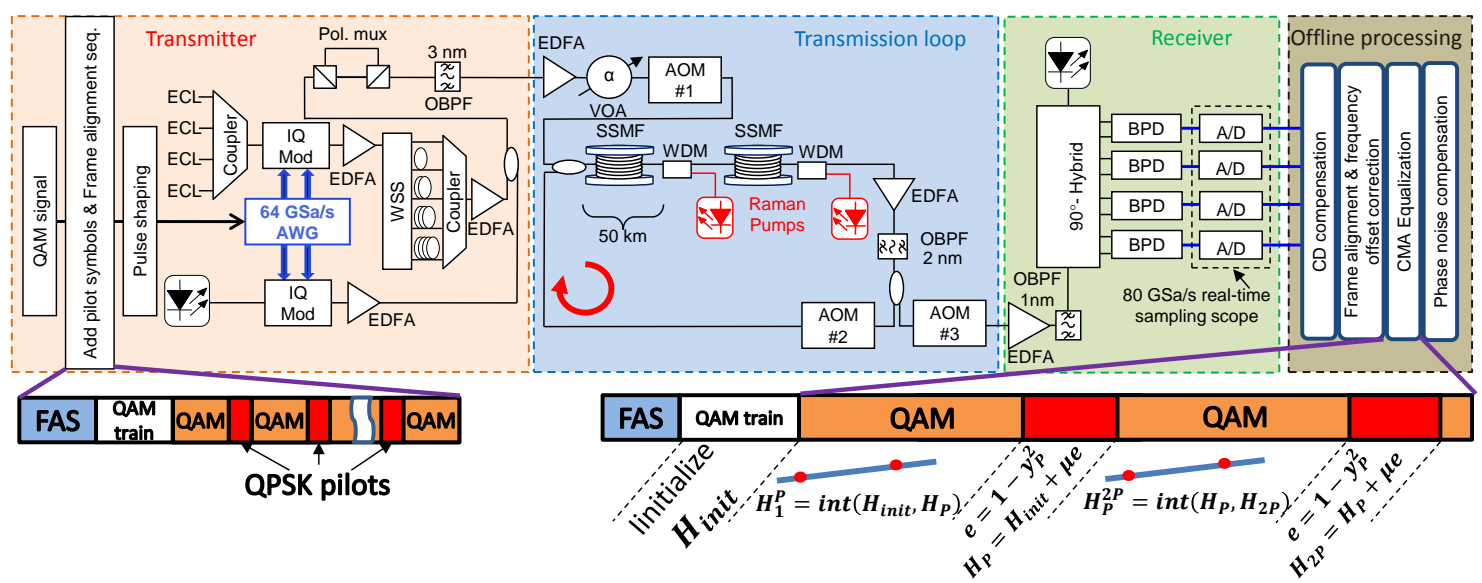

Fig. 1: Experimental setup and studied equalization. Five 10 GBaud channels with QAM are transmitted over a Raman-amplified recirculating loop, then detected by $80 \mathrm{GSa} / \mathrm{s}$ receiver and processed offline. QPSK pilots are interleaved with the QAM signal. The equalizer taps are found for the pilot positions only, and then interpolated to equalize the entire QAM sequence.

where $(k+1) P$ is the length of the block (in our case, we fix the QAM sequence length to $k \cdot(P-1)=60000$ ). In this work, the equalizer filter length is 101 symbols at 2 samples per symbol.

The experimental setup is shown in Fig. 1. At the transmitter, the pulse-shaped (raised cosine, roll-off 0.5) sequence (QAM + pilots) is sent to a $64 \mathrm{GSa} / \mathrm{s}$ arbitrary waveform generator (AWG), which is used to modulate five channels on a $25 \mathrm{GHz}$ grid at $10 \mathrm{GBaud}$ with two IQ modulators (for channel under test (CUT) and interfering channels).

The CUT (in this work that is the central channel) uses a sub-kHz linewidth fiber laser (Koheras BasiK C-15) while the four co-propagating channels use standard external cavity lasers (ECL, $100 \mathrm{kHz}$ linewidth). These four channels are decorrelated by more than 40 symbols using a wavelength selective switch (WSS) and combined with the CUT. A delay-and-add polarization emulator provides a dual-polarization signal, which is transmitted over a recirculating loop with $100 \mathrm{~km}$ loop length using distributed Raman amplification (DRA) with backward pumping every $50 \mathrm{~km}$. The signal is detected by an $80 \mathrm{GSa} / \mathrm{s}$ coherent receiver followed by offline processing, consisting of (in order) lowpass filtering, down sampling, chromatic dispersion (CD) compensation, frame alignment, frequency offset estimation, time-domain equalization and carrier phase recovery with the algorithm from [7].

\section{Results}

We study the performance of 256/1024QAM for up to $1600 \mathrm{~km}$ transmission, where PMD is accumulated $(<$ $1 \mathrm{ps} / \sqrt{\mathrm{km}}$ ), which strengthens the adaptation speed requirement of the equalizer. The received signal-to-noise ratio (SNR) and the achievable information rate are reported. The SNR is defined as SNR $=\mathbb{E}_{k}\left[\left|x_{k}\right|^{2}\right] / \mathbb{E}_{k}\left[\left|y_{k}-x_{k}\right|^{2}\right]$, where $y_{k}$ are the received samples after carrier phase recovery and $x_{k}$ are the transmitted QAM symbols at time $k$. The AIR is the mutual information (MI) $\mathscr{I}(X ; Y)$ between $x$ and $y$ assuming circular symmetric Gaussian statistics of the noise. While it is clear that higher PO improves the receiver SNR, the net data rate is reduced, which is indicated in the expression for MI: $\mathscr{I}(X ; Y)=(1-1 / P) \mathscr{H}(X)-\mathscr{H}(X \mid Y)$, where $\mathscr{H}$ is the entropy function. Higher PO improves the quality of the signal and reduces the uncertainty around the input given the output $(\mathscr{H}(X \mid Y))$, but at the same time reduces the entropy of the input $\mathscr{H}(X)$, resulting in a non-trivial trade-off.

The results are given in Figs. 2(a), 2(b), 2(c) and 2(d) for uniform 256QAM, probabilistically shaped 256QAM, uniform 1024QAM and probabilistically shaped 1024QAM. The probabilistic shaping is performed as in [5], and further details are omitted here. We compare the received SNR to the upper bound, achieved by a genie-aided MMA, in which the true transmitted symbols $x$ are used for equalization, i.e. all decisions are correct.

For all modulation formats, around $10 \%$ pilots are needed to converge to the upper bound (all modulation formats achieve virtually equivalent SNR at each pilot rate). However, the AIR is significantly reduced by such high PO. The optimal pilot rate is found to be between $2 \%$ and $5 \%$ for all modulation formats, resulting in modulation formatindependent performance. The effective SNR at the receiver does not allow for standard RDE MMA equalization due to the high symbol error rate, whereas the studied method is SNR-independent. We note that the received SNR is relatively low for the large modulation formats considered here. Such operating points are of interest since higher FEC overheads can be used and thus a better spectral efficiency can be achieved, as explained in the introduction.

Discussion. The complexity of the algorithm is small due to the simplicity of CMA and the fact that the taps 

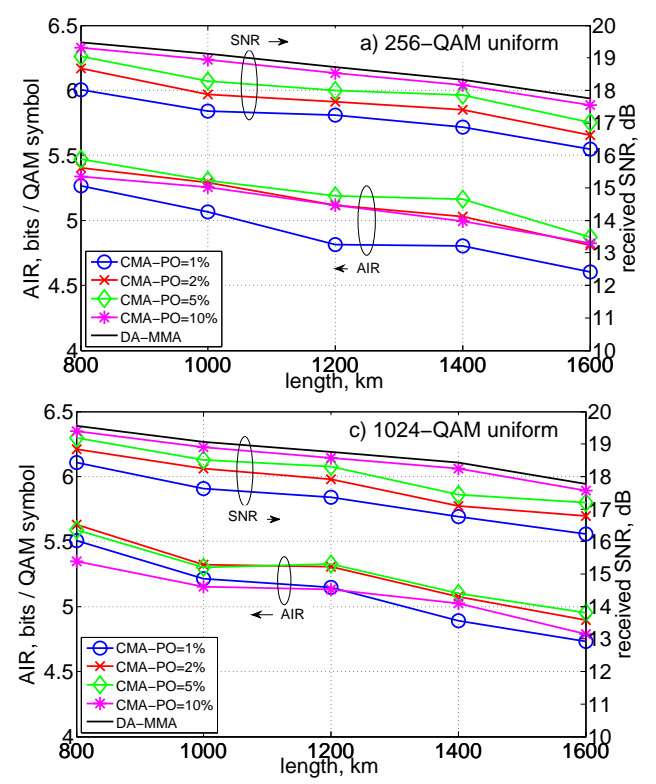

Fig. 2: Performance of the pilot-based equalization in a WDM system with 256/1024QAM with uniform and probabilistic shaping as in [5].

are only updated every $P$ symbols. Thus, $P$ times less complexity is achieved w.r.t. standard CMA (neglecting the complexity of the linear interpolation), and even more so w.r.t. RDE MMA. The algorithm also provides a flexible complexity/performance trade-off by tuning the PO. Since the equalization is only performed on the amplitude of the pilots, data can be transmitted on those positions using QPSK constellation with 2 bits/symbol efficiency, leading to a hybrid modulation format with extreme benefits (the equalizer comes for free). An alternative is to use the pilots during carrier phase recovery, including frequency offset and laser phase noise (as in [4-6]), in which case the pilots should not carry data. Blind carrier phase recovery can also take advantage of pilots for cycle-slip detection. Finally, we note that $\mathrm{PO}$ of $1 \%$ is used in e.g. [6], which is sufficient for back-to-back equalization of 64QAM. As we see in Fig. 2, fiber impairments (e.g. PMD) result in a fast-varying channel, and thus introduce the need for higher PO.

\section{Conclusion}

Pilot-based equalization was experimentally studied for WDM systems with high-order QAM and probabilistic shaping. Modulation format-independent performance was reported over metro-range distances $(800-1600 \mathrm{~km})$ with an optimized pilot insertion rate of between $2 \%$ and $5 \%$. The equalization scheme allows for operation at low received SNR relative to the modulation format size and with higher FEC overhead, thus with a better spectral efficiency at a given distance.

\section{Acknowledgments}

This work was supported by the DNRF Research Centre of Excellence, SPOC, ref. DNRF123. NKT Photonics A/S is acknowledged for providing the narrow linewidth fiber lasers.

\section{References}

1. S. Savory, "Digital signal processing for multilevel modulation formats" in ECOC, p. Tu.2.A.1, (2016).

2. I. Fatadin, et. al., "Blind equalization and carrier phase recovery in a 16-QAM optical coherent system", JLT, 27(15), pp. 3042-3049, (2009).

3. F. Buchali, et. al., "Rate adaptation and reach increase by probabilistically shaped 64-QAM: an experimental demonstration", JLT, 34(7), pp. 1599-1609(2016).

4. M. P. Yankov, et. al., "Constellation shaping for fiber-optic channels with QAM and high spectral efficiency", Photon. Techn. Lett., 26(23), pp. 2407-2410, (2014).

5. M. P. Yankov, et. al., "Constellation shaping for WDM systems using 256QAM/1024QAM with probabilistic optimization", JLT, DOI:10.1109/JLT..2016.2607798, (2016).

6. D. S. Millar, et. al., "Design of a $1 \mathrm{~Tb} / \mathrm{s}$ superchannel coherent receiver", JLT, 34(6), pp. 1453-1463, (2016).

7. M. P. Yankov, et. al., "Low-complexity tracking of laser and nonlinear phase noise in WDM optical fiber systems”, JLT, 33(23), 4975-4984(2015). 